LMU-ASC $61 / 05$

MPP-2005-94

hep-th/0509082

\title{
Fermion Zero Modes in the Presence of Fluxes and a Non-perturbative Superpotential
}

\author{
Dieter Lüst ${ }^{a, b, 1}$, Susanne Reffert ${ }^{b, 2}$, Waldemar Schulgin ${ }^{b, 3}$ and Prasanta K. Tripathy ${ }^{a, 4}$ \\ ${ }^{a}$ Arnold-Sommerfeld-Center for Theoretical Physics \\ Department für Physik, Ludwig-Maximilians-Universität München \\ Theresienstraße 37, 80333 München, Germany \\ ${ }^{b}$ Max-Planck-Institut für Physik, Föhringer Ring 6, 80805 München, Germany
}

\begin{abstract}
We study the effect of background fluxes of general Hodge type on the supersymmetry conditions and on the fermionic zero modes on the world-volume of a Euclidean $M 5 / D 3$-brane in $M$-theory/type $I I B$ string theory.

Using the naive susy variation of the modulino fields to determine the number of zero modes in the presence of a flux of general Hodge type, an inconsistency appears. This inconsistency is resolved by a modification of the supersymmetry variation of the modulinos, which captures the back-reaction of the non-perturbative effects on the background flux and the geometry.
\end{abstract}

\footnotetext{
${ }^{1}$ luest@mppmu.mpg.de, luest@theorie.physik.uni-muenchen.de

${ }^{2}$ sreffert@theorie.physik.uni-muenchen.de

${ }^{3}$ schulgin@theorie.physik.uni-muenchen.de

${ }^{4}$ prasanta@theorie.physik.uni-muenchen.de
} 


\section{Introduction and Summary}

Recently, there has been a lot of progress in the investigation of KKLT-type models [1]. On the one hand, specific examples of candidate models have been constructed [2, 3]. On the other hand, the generation of a non-perturbative superpotential which may serve to stabilize all Kähler moduli has been investigated in much detail. The recent research in this line extends the earlier work of Witten [4] by taking into account non-vanishing background fluxes $[5,6,7,8,9,10]$ and working out the conditions for the generation of the superpotential directly for type $I I B$-orientifolds without the detour of analyzing the $M / F$-theory case first $[11,12,13]$. If $M 5 / D 3$-brane instantons wrapping a divisor in the compactification manifold are the source of a possible non-perturbative superpotential, the analysis involves deriving the Dirac equation in the world-volume of the $M 5 / D 3_{-}$ brane and studying the structure of its fermionic zero modes. So far, only the case of the background flux being of Hodge type $(2,2)$ in $M / F$-theory, or $(2,1)$ in type $I I B$-theory has been considered.

The present letter resolves a seeming puzzle concerning the fermionic zero mode structure in the presence of background fluxes of general Hodge type. As has been shown in $[14,15]$, the conditions for a supersymmetric background flux obtained from the minimization of the effective four-dimensional superpotential change in the presence of a non-perturbative term. The supersymmetric flux is no longer of Hodge type $(2,2)$ (resp. $(2,1)$ for type $I I B)$, but receives contributions of all Hodge types. We will show that, if one now, guided by this result, plugs a flux of general Hodge type into the zero mode conditions obtained from the Dirac equation, an inconsistency arises: If with $(2,2)$-flux, the conditions for the generation of a superpotential were met, this is no longer the case for general flux.

As we explain in the following, this apparent mismatch disappears after the introduction of a modification of the supersymmetry variation of the modulino, which basically captures the back-reaction of the non-perturbative effects on the background flux and the geometry.

This paper is organized as follows. In section 2, we discuss the supersymmetry conditions on the background flux as obtained from the effective potential as well as from the supersymmetry variation of the modulino. In section 3 , the fermionic zero modes on the world-volume of $D 3 / M 5$-branes are analyzed. First, we study the conditions on the zero modes originating from the Dirac equation in the presence of fluxes of general Hodge type. We find a modification from the case of pure $(2,2)$, (respectively $(2,1))$ flux. To further elucidate the effect of allowing general background fluxes, the concrete exam- 
ple of compactification of $M / F$-theory on $K 3 \times K 3$ is presented. If a pure $(2,2)-$ flux is turned on, a non-perturbative superpotential is generated. If, on the other hand, we allow other Hodge components, which a supersymmetric flux solution in the presence of a nonperturbative superpotential requires, all zero modes are lifted and no non-perturbative superpotential is generated. Thus, an obvious inconsistency arises.

In section 4, we set out to resolve the puzzle. We find that the non-perturbative superpotential must be included into the susy variation of the 11-dimensional gravitino field after compactification, which in turn determines the Dirac equation and therefore the number of zero modes. Like this, the $(4,0)$ - and $(3,1)$-parts of the flux are balanced by the contribution from the non-perturbative superpotential, and the number of zero modes remains the same as for $(2,2)$-flux.

\section{The supersymmetry conditions}

In this section, we will study the supersymmetry conditions for the low energy theory arising from string compactifications in the presence of background fluxes and due to non-peturbative effects. We will first obtain the susy conditions by minimizing the effective potential and then by analyzing the spinor conditions. We work out the case for IIB compactification on Calabi-Yau threefolds and then briefly discuss $M$-theory compactification on Calabi-Yau fourfolds.

\subsection{Effective Potential}

We first consider the compactification of type IIB theory on a Calabi-Yau threefold. The resulting low energy supergravity action is given by

$$
S=\int d^{4} x \frac{1}{2} \sqrt{-g}\left\{R+g_{A \bar{B}} \partial_{\mu} z^{A} \partial^{\mu} \bar{z}^{B}\right\}+V_{\text {eff }}+S_{\text {gauge }} .
$$

Here, we used a condensed notation: The indices $\{A, B, \cdots\}=\{i, I, \tau\}$ denote both the complex structure moduli $\{i\}$, Kähler moduli $\{I\}$, and the complexified axion-dilaton field $\tau$. $S_{\text {gauge }}$ denotes the gauge field dependent part of the action. The effective potential

$$
V_{e f f}=\frac{1}{2} e^{K}\left(g^{A B} D_{A} W \overline{D_{B} W}-3|W|^{2}\right)
$$

is given in terms of the total superpotential

$$
W=W_{f l u x}+W_{n p}
$$


and the Kähler potential $K$. Here $W_{f l u x}$ is the flux superpotential [16]

$$
W_{f l u x}=\int G_{3} \wedge \Omega_{3}
$$

and $W_{n p}$ is the superpotential arising from nonperturbative effects. $\Omega_{3}$ is the holomorphic $(3,0)$-form on the CY space and

$$
G_{3}=F_{3}-\tau H_{3}
$$

$F_{3}$ and $H_{3}$ being the RR and NS field strengths, respectively. The flux superpotential depends only on the complex structure moduli. We assume the nonperturbative superpotential to depend on the Kähler moduli only.

The supersymmetry preserving minima are obtained by solving the equations

$$
D_{A} W=0
$$

It is well known that in the absence of a nonperturbative term, $W=W_{f l u x}$, the condition (2.6) requires $G_{3}$ to be of type $(2,1)$ and primitive [17]. For $W_{n p} \neq 0$, this is no longer true [15], and $G_{3}$ acquires non-vanishing $(1,2),(3,0)$ and $(0,3)$ parts:

$$
\begin{aligned}
& \int G_{3} \wedge \chi_{i}^{(2,1)}+\partial_{i} K W_{n p}=0, \\
& \int G_{3} \wedge \Omega_{3} \partial_{I} K+D_{I} W_{n p}=0, \\
& \int \bar{G}_{3} \wedge \Omega_{3}+W_{n p}=0 .
\end{aligned}
$$

The primitivity condition $G_{3} \wedge J=0$, being a $D$-term condition, remains intact despite $W_{n p}$. Here $\chi_{i}^{(2,1)}$ is a form of type $(2,1)$.

We can similarly obtain the susy conditions for M-theory compactification on a CalabiYau fourfold. The flux superpotential is now given by [18]

$$
W_{\text {flux }}=\int G_{4} \wedge \Omega_{4}
$$

Here, $G_{4}$ is the four-form flux present in 11-dim. supergravity theory and $\Omega_{4}$ is the holomorphic $(4,0)$-form on the CY fourfold. The susy conditions take the form:

$$
\begin{aligned}
& \int G_{4} \wedge \chi_{i}^{(3,1)}+\partial_{i} K W_{n p}=0, \\
& \int G_{4} \wedge \Omega_{4} \partial_{I} K+D_{I} W_{n p}=0 .
\end{aligned}
$$

In the following subsection, we will show how the above conditions can be derived from the modulino variations. 


\subsection{Spinor Conditions}

Now, it is important to remember that the BPS susy variation of the gravitino is equivalent to solving the susy conditions in the effective field theory, as discussed in [19] for M-theory on a fourfold, in [20] and in [21] for type IIB on a CY threefold, and also by [22] for the heterotic string. Thus we must modify the spinor conditions accordingly in order to obtain the susy conditions eq.(2.7) in $I I B$ theory and eq.(2.9) in M-theory. In what follows, we will first review the spinor conditions in the absence of $W_{n p}$, and then consider the generalization when $W_{n p}$ is included.

Let us first consider the situation in $I I B$ theory. This has been worked out in [21]. The supersymmetry variations can be summarized as follows:

$$
\begin{aligned}
\kappa \delta \psi_{\mu} & =\partial_{\mu} \epsilon-\frac{1}{8} \gamma_{\mu} \gamma^{m}\left(\partial_{m} \ln Z-4 \kappa Z \Gamma^{4} \partial_{m} h\right) \epsilon+\frac{1}{16} \kappa \gamma_{\mu} G \epsilon^{*} \\
\kappa \delta \psi_{m} & =\left(\tilde{D}_{m}-\frac{i}{2} Q_{m}\right) \epsilon+\frac{1}{8} \epsilon \partial_{m} \ln Z-\frac{1}{16} \kappa \gamma_{m} G \epsilon^{*}-\frac{1}{8} \kappa G \gamma_{m} \epsilon^{*} \\
\kappa \delta \lambda^{*} & =-i \gamma^{m} P_{m}^{*} \epsilon+\frac{i}{4} \kappa \bar{G} \epsilon^{*}
\end{aligned}
$$

The first equation is the supersymmetry variation of the four-dimensional gravitino field. Second, $\delta \psi_{m}$ corresponds to the variation of the internal gravitino. After compactification the internal gravitino degrees of freedom become in the effective 4D field theory the modulino fields, i.e. the fermionic superpartners of the Kähler and complex structure moduli fields. Concretely, the modulino equations which one obtains by dimesional reduction (see appendix) are

$$
\begin{array}{rlrl}
\delta \phi_{e \overline{a b}}^{i} & =-\frac{1}{8} G_{e a b}^{i} \hat{\xi}^{*}-\frac{1}{16} g^{a \bar{c}} g_{e \bar{a}} G_{a \overline{b c}}^{i} \hat{\xi}^{*}, & & i=1, \ldots, h^{(2,1)}, \\
\delta \phi_{\bar{e} \mid \overline{a b}}^{I} & =-\frac{1}{16} G_{\overline{e a b}} \hat{\xi}^{*}, & & I=1, \ldots, h^{(1,1)} \\
\delta \lambda_{\overline{a b c}}^{*} & =\frac{i}{4} \bar{G} \overline{a b c} \hat{\xi}^{*} &
\end{array}
$$

where $\hat{\xi}$ is a four dimensional supersymmetry parameter. Finally, $\delta \psi_{m}$ indeed comprises the supersymmetry variations of all modulinos, namely it leads after compactification to $h_{1,1}+h_{2,1}$ independent spinor equations, which we call modulino equations. Finally, $\delta \lambda^{*}$ is the supersymmetry variation of the four-dimensional dilatino. In these equations, we use the same notation as [21]. In particular, $G=\frac{1}{6} G_{m n p} \gamma^{m n p}, Z$ is the warp factor, $\tilde{D}_{m}$ is the covariant derivative with respect to the internal metric, $h$ is related to the $\mathrm{RR}$ four-form field, $h=C_{0123}$, and

$$
\begin{aligned}
& P_{m}=f^{2} \partial_{m} B, \quad Q_{m}=f^{2} \operatorname{Im}\left(B \partial_{m} B^{*}\right) \\
& B=\frac{1+i \tau}{1-i \tau}, \quad f^{-2}=1-B B^{*} .
\end{aligned}
$$


The conditions $(2.10)$ can be solved to show that $G_{3}$ is of type $(2,1)$ and primitive.

Clearly, the explicit dependence on the superpotential $W_{\text {flux }}$ and its covariant derivatives is not apparent in the modulino variations (2.11). We need to make this precise, in order to generalize the above formulae in presence of $W_{n p}$. Since we are interested in the $G_{3}$ dependence of the variations, we can as well ignore the effects of warping and the five-form flux, and also set the complexified axion-dilaton field to constant.

It is now easy to introduce the flux superpotential in the above equations. Note that

$$
\begin{aligned}
& D_{i} W_{\text {flux }}=\int G_{3} \wedge \chi_{i}^{2,1} \Longrightarrow G_{a \overline{b c}}^{i}=\epsilon_{a \overline{b c}} D_{i} W_{f l u x} \\
& D_{I} W_{\text {flux }}=\partial_{I} K \int G_{3} \wedge \Omega_{3} \Longrightarrow G_{\overline{a b c}}=\epsilon_{\overline{a b c}} \frac{D_{I} W_{\text {flux }}}{\partial_{I} K}
\end{aligned}
$$

Substituting the above into the modulino variations, we find

$$
\begin{array}{rlrl}
\delta \phi_{e \bar{e} \bar{b}}^{i} & =-\frac{1}{8} \epsilon_{e \overline{e a b}} D_{i} W_{\text {flux }}-\frac{1}{16} g^{a \bar{c}} g_{e \bar{a}} G_{a \overline{a b c}}^{i} \hat{\xi}, & i=1 \ldots h^{(2,1)}, \\
\delta \phi_{\bar{e} \mid \overline{a b}}^{I}=-\frac{1}{16} \epsilon_{\overline{e a b}} \frac{D_{I} W_{\text {flux }}}{\partial_{I} K}, & I=1 \ldots h^{(1,1)} .
\end{array}
$$

Similarly, using

$$
\bar{G} \overline{a b c}=-\epsilon \overline{a b c}(\tau-\bar{\tau}) D_{\tau} W_{f l u x}
$$

we find

$$
\delta \lambda_{a b c}^{*}=-\frac{i}{4} \epsilon \frac{}{a b c}(\tau-\bar{\tau}) D_{\tau} W_{f l u x} \hat{\xi}
$$

For covariantly constant spinors, we recover the susy conditions

$$
D_{i} W_{\text {flux }}=D_{I} W_{\text {flux }}=D_{\tau} W_{\text {flux }}=0
$$

Now, it is easy to generalize the spinor variations in presence of the non-perturbative superpotential. We simply replace $W_{f l u x}$ by $W=W_{\text {flux }}+W_{n p}$. The variation equations then become

$$
\begin{aligned}
\delta \phi_{e \overline{a b}}^{i} & =-\frac{1}{8} \epsilon_{e \overline{b c}} D_{i} W-\frac{1}{16} g^{a \bar{c}} g_{e \bar{a}} G_{a \overline{a c}}^{i} \hat{\xi}, \\
\delta \phi_{\bar{e} \mid \overline{a b}}^{I} & =-\frac{1}{16} \epsilon_{\overline{e a b}} \frac{D_{I} W}{\partial_{I} K}, \\
\delta \lambda_{\overline{a b c}}^{*} & =-\frac{i}{4} \epsilon \overline{a b c}(\tau-\bar{\tau}) D_{\tau} W .
\end{aligned}
$$

We clearly see that, for covariantly constant spinors, the first of the above equations implies the flux to be primitive and in addition $D_{i} W$ is zero. The second and third equations then imply that $D_{I} W$ and $D_{\tau} W$ are zero respectively. Thus we recover the susy conditions

$$
D_{i} W=D_{I} W=D_{\tau} W=0 .
$$


We now proceed to work out the modulino transformations in M-theory in presence of $W_{n p}$ in a similar fashion. This has been analyzed in [19]. We will first express the variation equations in terms of the flux superpotential, and then generalize it to the case of $W_{n p} \neq 0$. Consider first the internal gravitino variation without $W_{n p}$ :

$$
\delta \psi_{m}=\nabla_{m} \xi+\frac{1}{24} \gamma^{n p q} G_{m n p q} \xi
$$

By dimensional reduction we obtain (see appendix)

$$
\begin{aligned}
\delta \phi_{e \bar{c}}^{k} & =\frac{1}{4}\left(G_{e b \overline{c d}} g^{b \bar{d}}\right)^{k} \hat{\xi}, & k & =1, \ldots, h^{(1,1)}, \\
\delta \phi_{e \overline{a b c}}^{i} & =\frac{1}{24} G_{e a b c}^{I} \hat{\xi}, & i & =1, \ldots, h^{(3,1)}, \\
\delta \phi_{\bar{e} \mid \overline{a b c}}^{I} & =\frac{1}{24} G_{\overline{e a b c} \hat{\xi}} \hat{\xi} & I & =1, \ldots, h^{(1,1)} .
\end{aligned}
$$

By solving the susy conditions, we get in general $h_{3,1}$ equations for the complex structure moduli and $h_{1,1}$ equations for the Kähler moduli. The same conditions should be reproduced by setting $\delta \phi^{i}$ and $\delta \phi^{I}$ to zero. There are $h^{3,1}$ fluxes of type $(1,3)$. The $(0,4)$-flux is a solution of $h^{1,1}$ independent equations. Because of these reasons, it is natural to say that for every $G_{a \overline{b c d}}$ and every $G_{\overline{a b c d}}$ (same $G_{\overline{a b c d}}$ coming from $h^{1,1}$ equations), the variation of the gravitino should be zero.

There is no $I$ on the r.h.s. This emphasizes the fact that the $h^{1,1}$ supersymmetry conditions are degenerate in the $(0,4)$-flux. Using

$$
D_{i} W_{\text {flux }}=\int G_{4} \wedge \chi_{3,1}^{i}=G_{e \overline{b c d}}^{i} \epsilon^{e \overline{b c d}}
$$

and

$$
D_{I} W_{\text {flux }}=\partial_{I} K \int G_{4} \wedge \Omega_{4}=\partial_{I} K G_{\overline{e b c d}} \epsilon^{\overline{e b c d}}
$$

we can immediately rewrite (2.21) into

$$
\begin{array}{rlrl}
\delta \phi_{e \bar{c}}^{k} & =\frac{1}{4}\left(G_{e b \overline{c d}} g^{b \bar{d}}\right)^{k} \hat{\xi}, & & k=1, \ldots, h^{(1,1)}, \\
\delta \phi_{e \overline{e a b c}}^{i} & =\frac{1}{24} \epsilon_{e \overline{e a b c}} D_{i} W_{f l u x} \hat{\xi}, & & i=1, \ldots, h^{(3,1)} \\
\delta \phi_{\bar{e} \mid \overline{a b c}}^{I} & =\frac{1}{24} \epsilon_{\overline{e a b c}} \frac{D_{I} W_{\text {flux }} \hat{\xi},}{\partial_{I} K} & I=1, \ldots, h^{(1,1)} .
\end{array}
$$

The supersymmetry conditions and the primitivity condition are reproduced by setting $\delta \phi^{k}, \delta \phi^{i}, \delta \phi^{I}$ to zero.

This gives immediately

$$
g^{a \bar{d}} g^{b \bar{c}} G_{e b \overline{c d}}=0
$$




$$
\begin{aligned}
& D_{i} W_{\text {flux }}=0, i=1, \ldots, h^{1,3} \\
& D_{I} W_{\text {flux }}=0, I=1, \ldots, h^{1,1} .
\end{aligned}
$$

These equations correspond to the primitivity conditions on $G_{2,2}$ and the vanishing of $G_{1,3}$ and $G_{0,4}$.

In the next step, we would like to make a proposal for the form of the additional terms of the supersymmetry variation of the modulinos in the presence of the non-perturbative term $W_{n p}$. The supersymmetry conditions which should be reproduced, change to

$$
\begin{aligned}
& D_{i} W=D_{i} W_{\text {flux }}+D_{i} W_{n p}=0, \\
& D_{I} W=D_{I} W_{\text {flux }}+D_{I} W_{n p}=0 .
\end{aligned}
$$

From (2.24), we immediately see that the variation of the modulinos should be changed to

$$
\begin{array}{rlrl}
\delta \phi_{e \bar{c}}^{k} & =\frac{1}{4}\left(G_{e b \overline{c d}} g^{b \bar{d}}\right)^{k} \hat{\xi}, & k=1, \ldots, h^{(1,1)}, \\
\delta \phi_{e \overline{e a b c}}^{i}=\frac{1}{24} \epsilon_{e \overline{e a b c}} D_{i} W \hat{\xi}, & i=1, \ldots, h^{(3,1)}, \\
\delta \phi_{\bar{e} \mid \overline{a b c}}^{I}=\frac{1}{24} \epsilon_{\overline{e a b c}} \frac{D_{I} W}{\partial_{I} K} \hat{\xi}, & I=1, \ldots, h^{(1,1)} .
\end{array}
$$

\section{Conditions on the zero modes from fluxes and the non-perturbative superpotential}

The non-perturbative superpotential may be generated via gaugino condensation or via instanton effects or both. Here, we will concentrate on the case of instantons. In type II B theory, they correspond to Euclidean D3-branes wrapping divisors of the CY threefold, whereas in M-theory, they come from Euclidean $M 5$-branes wrapping divisors of the CY fourfold. It has been pointed out by Witten [4] some time ago that the necessary condition for an M5-instanton to generate a superpotential is that the corresponding divisor has holomorphic Euler characteristic equal to one. This provides a stringent condition on the possible CY fourfolds [23]. For type II B compactification on a CalabiYau without the orientifold projection (without flux), the index is always zero and hence no superpotential is generated due to instanton effects [11]. It has been argued recently [24], that the index might change in the presence of flux. An explicit example has been constructed to show that some of the wold-volume fermion zero modes are lifted due to flux [6]. Subsequently, a generalized index formula was derived in M-theory [7, 8], as well

as in type $I I B$ theory [11]. However, these results are based on the assumption that the 
flux is primitive and of type $(2,1)$ in type $I I B$, or $(2,2)$ respectively in M-theory. As we have already discussed, the supersymmetric flux no longer remains $(2,1)$ (resp. $(2,2)$ ) in presence of the non-perturbative superpotential. In this section, we will analyze the fermion zero modes on the world volume of $D 3 / M 5$-branes in the presence of general flux.

\subsection{General fluxes}

The fermionic bilinear terms in the D3-brane world-volume action in presence of background flux have been derived in $[25,6]$ by using the method of gauge completion, and also in $[26,27,9]$ from the $M 2$-brane world volume action using T-duality. Upon Euclidean continuation and by an appropriate gauge choice [11], the Lagrangian takes the form

$$
L^{D 3}=2 \sqrt{\operatorname{det} g} \theta\left\{e^{-\phi} \gamma^{m} \nabla_{m}+\frac{1}{8} \tilde{G}_{m n \hat{p}} \gamma^{m n \hat{p}}\right\} \theta
$$

Here $m, n, \ldots$ are directions along the brane and $\hat{p}$ stands for directions transverse to the brane. As always, we turn on the three-form flux only along the directions of the internal manifold. Also for simplicity, we set the flux $F_{2}$ due to the world-volume gauge fields to zero. $\tilde{G}$ is defined to be

$$
\tilde{G}_{m n p}=e^{-\phi} H_{m n p}+i F_{m n p}^{\prime} \gamma_{5}
$$

with $F^{\prime}=d C_{2}-C_{0} H_{3}$. The Dirac equation, obtained from the above action, reads

$$
\left\{e^{-\phi} \gamma^{m} \nabla_{m}+\frac{1}{8} \tilde{G}_{m n \hat{p}} \gamma^{m n \hat{p}}\right\} \theta=0
$$

Locally, we can express the internal metric as

$$
d s^{2}=g_{a \bar{b}} d y^{a} d y^{\bar{b}}+g_{z \bar{z}} d z d \bar{z}
$$

where $a, b, \ldots$ are complex coordinates on the $D 3$-brane and $z, \bar{z}$ are directions transverse to the brane. We define the Clifford vacuum to be

$$
\gamma^{z}\left|\Omega>=\gamma^{a}\right| \Omega>=0
$$

The spinor $\theta$ can be written in terms of positive and negative chirality spinors as $\theta=$ $\epsilon_{+}+\epsilon_{-}$with

$$
\begin{aligned}
& \epsilon_{+}=\phi\left|\Omega>+\phi_{\bar{a}} \gamma^{\bar{a}}\right| \Omega>+\phi_{\overline{a b}} \gamma^{\overline{a b}} \mid \Omega> \\
& \epsilon_{-}=\phi_{\bar{z}} \gamma^{\bar{z}}\left|\Omega>+\phi_{\overline{a z}} \gamma^{\overline{a z}}\right| \Omega>+\phi_{\overline{a b z}} \gamma^{\overline{a b z}} \mid \Omega>.
\end{aligned}
$$


Substituting this into the Dirac equation, we find

$$
\begin{array}{r}
e^{-\phi} 2 g^{a \bar{a}} \partial_{a} \phi_{\bar{a}}+2 i g^{z \bar{z}} g^{a \overline{b^{\prime}}} g^{b \overline{a^{\prime}}} G_{a b z} \phi_{\overline{a^{\prime} b^{\prime} z}}+\frac{1}{2} i g^{z \bar{z}} g^{a \bar{b}} \phi_{\bar{z}} G_{a \bar{b} z}=0, \\
e^{-\phi}\left(\partial_{\overline{a^{\prime}}} \phi+4 g^{a \overline{b^{\prime}}} \partial_{a} \phi_{\overline{b^{\prime} a^{\prime}}}\right)+\frac{1}{2} i g^{z \bar{z}} g^{a \bar{b}}\left(\phi_{\overline{a^{\prime} z}} \bar{G}_{a \bar{b} z}-2 \phi_{\overline{b z}} \bar{G}_{a \bar{a} z}\right)=0 \\
e^{-\phi} \partial_{\left[\overline{a^{\prime}}\right.} \phi_{\left.\overline{b^{\prime}}\right]}+\frac{1}{2} i g^{z \bar{z}} g^{a \bar{b}}\left(\phi_{\overline{a^{\prime} b^{\prime} z}} G_{a \bar{b} z}-4 \phi_{\overline{b^{\prime} z}} G_{a \bar{a}^{\prime} z}\right)+\frac{1}{4} i g^{z \bar{z}} \phi_{\bar{z}} G_{\overline{a^{\prime} b^{\prime}} z}=0
\end{array}
$$

and

$$
\begin{array}{r}
e^{-\phi} 2 g^{a \bar{a}} \partial_{a} \phi_{\overline{a z}}+i g^{a \overline{b^{\prime}}} g^{b \overline{a^{\prime}}} \phi_{\overline{a^{\prime} b^{\prime}}} G_{a b \bar{z}}+\frac{1}{4} i g^{a \bar{b}} \phi G_{a \overline{b z}}=0, \\
e^{-\phi}\left(\partial_{\overline{a^{\prime}}} \phi_{\bar{z}}+4 g^{a \overline{b^{\prime}}} \partial_{a} \phi_{\overline{b^{\prime} a^{\prime} z}}\right)-\frac{1}{4} i g^{a \bar{b}}\left(\phi_{\overline{a^{\prime}}} \bar{G}_{a \overline{b z}}-2 \phi_{\bar{b}} \bar{G} \bar{a} \overline{a^{\prime} z}\right) \\
e^{-\phi} \partial_{\left[\overline{a^{\prime}}\right.} \phi_{\overline{\left.b^{\prime}\right] z}}+\frac{1}{4} i g^{a \bar{b}}\left(\phi_{\overline{a^{\prime} b^{\prime}}} G_{a \overline{b z}}-4 \phi_{\overline{b b^{\prime}}} G_{a a^{\prime} z}\right)+\frac{1}{8} i \phi G_{\overline{a^{\prime} b^{\prime} z}}=0 .
\end{array}
$$

We can similarly work out the equations for world-volume M5-brane fermions. The fermionic bilinear terms on the $M 5$-brane world-volume in the presence of background flux have been derived in [5]. Upon setting the world volume gauge flux to zero, we have the Dirac equation

$$
\gamma^{m} \nabla_{m} \theta-\frac{1}{24} \gamma^{\hat{q}} \gamma^{m n p} G_{m n p \hat{q}} \theta=0
$$

Again, we turn on the fluxes only along the compact directions. Here, $m, n, p, \ldots$ are real indices. $\mathrm{A}^{\wedge}$ ' indicates the directions transverse to the brane. We denote by $a, b, \ldots$ the holomorphic indices along the brane and by $\bar{a}, \bar{b}, \ldots$ the anti-holomorphic indices; $z$ is the complex coordinate along the normal to the divisor. The spinor $\theta$ can be expressed in terms of the Clifford vacuum and the creation operators as

$$
\theta=\phi\left|\Omega>+\phi_{\bar{z}} \gamma^{\bar{z}}\right| \Omega>+\phi_{\bar{a} \bar{b}} \gamma^{\bar{a} \bar{b}}\left|\Omega>+\phi_{\bar{z} \bar{a} \bar{b}} \gamma^{\bar{z} \bar{a} \bar{b}}\right| \Omega>
$$

Plugging this expression for $\theta$ into the Dirac equation, we find

$$
\begin{aligned}
& \left(\partial_{\bar{c}} \phi+4 g^{b \bar{b}^{\prime}} \partial_{b} \phi_{\bar{b}^{\prime} \bar{c}}\right) \\
& +\frac{1}{2}\left[4 g^{a \bar{a}^{\prime}} g^{b \bar{b}^{\prime}} g^{z \bar{z}}\left(G_{a b \bar{b}^{\prime} z} \phi_{\bar{z} \bar{a}^{\prime} \bar{c}}-G_{a b \bar{c} z} \phi_{\bar{z} \bar{a}^{\prime} \bar{b}^{\prime}}\right)+g^{z \bar{z}} g^{a \bar{b}} \phi_{\bar{z}} G_{a \bar{b} \bar{c} z}\right]=0 \\
& \left(\partial_{\bar{a}} \phi_{\bar{z}}+4 g^{b \bar{b}^{\prime}} \partial_{b} \phi_{\bar{z} \bar{a} \bar{b}^{\prime}}\right) \\
& -\frac{1}{4}\left[4 g^{a \bar{a}^{\prime}} g^{b \bar{b}^{\prime}}\left(G_{a b \bar{b}^{\prime} \bar{z}} \phi_{\bar{a}^{\prime} \bar{c}}-G_{a b \bar{c} \bar{z}} \phi_{\bar{a}^{\prime} \bar{b}^{\prime}}\right)+g^{a \bar{b}} \phi G_{a \bar{b} \bar{c} \bar{z} \bar{z}}\right]=0 \\
& \partial_{[\bar{a} \bar{b}} \phi_{\bar{b} \bar{c}]}+\frac{1}{12} g^{z \bar{z}} \phi_{\bar{z}} G_{\bar{a} \bar{b} \bar{c} z}=0, \\
& \partial_{[\bar{a}} \phi_{\bar{z} \bar{b} \bar{c}]}+\frac{1}{24} \phi G_{\bar{a} \bar{b} \bar{c} \bar{z}}=0 .
\end{aligned}
$$

These expressions can be simplified a lot using the primitivity condition:

$$
\left(\partial_{\bar{c}} \phi+4 g^{b \bar{b}^{\prime}} \partial_{b} \phi_{\bar{b}^{\prime} \bar{c}}\right)-2 g^{a \bar{a}^{\prime}} g^{b \bar{b}^{\prime}} g^{z \bar{z}} G_{a b \bar{c} z} \phi_{\bar{z} \bar{a}^{\prime} \bar{b}^{\prime}}=0
$$




$$
\begin{aligned}
& \left(\partial_{\bar{a}} \phi_{\bar{z}}+4 g^{b \bar{b}^{\prime}} \partial_{b} \phi_{\bar{z} \bar{a} \bar{b}^{\prime}}\right)+g^{a \bar{a}^{\prime}} g^{b \bar{b}^{\prime}} G_{a b \bar{c} \bar{z}} \phi_{\bar{a}^{\prime} \bar{b}^{\prime}}=0, \\
& \partial_{[\bar{a}} \phi_{\bar{b} \bar{c}]}+\frac{1}{12} g^{z \bar{z}} \phi_{\bar{z}} G_{\bar{a} \bar{b} \bar{c} z}=0, \\
& \partial_{[\bar{a}} \phi_{\bar{z} \bar{b} \bar{c}]}+\frac{1}{24} \phi G_{\bar{a} \bar{b} \bar{c} \bar{z}}=0 .
\end{aligned}
$$

The equations are modified due to the $(3,1)$ - and $(4,0)$-fluxes, and so is the zero mode

counting. To understand this better, we shall turn to the example of compactification on $K 3 \times K 3$.

\subsection{Example: $K 3 \times K 3$}

To acquire a better understanding of the above equations, we consider here the example of M/F-theory compactified on $K 3_{1} \times K 3_{2}$ with background flux [29, 10, 30]. Consider one of the $K 3$ s (say $K 3_{2}$ ) to be elliptically fibered. Wrap the $M 5$-brane on one of the divisors of the form $K 3 \times S$, where $S$ corresponds to the $P^{1}$ s of the elliptic $K 3$. Let $z$ parametrize the direction normal to the brane.

We will now briefly review the case of the flux being of type $(2,2)$ and primitive and then consider the case of general flux. Let us first analyze the case of the flux preserving $N=2$ supersymmetry. In this case, the $(2,2)$-flux must take the form

$$
G_{4} \in H^{1,1}\left(K 3_{1}\right) \otimes H^{1,1}\left(K 3_{2}\right)
$$

which implies that the $N=2$ flux must be a $(1,1)$-form in $K 3_{2}$. Since it is an elliptically fibered $K 3$, we have to use the spectral sequence, which tells us that the flux belongs to $[28]$

$$
H^{0}\left(B, R^{2} \pi_{*} \mathbf{R}\right) \oplus H^{2}\left(B, \pi_{*} \mathbf{R}\right)
$$

which in simple terms means that the flux has either both legs in the fiber or both in the base. So the $N=2$ flux is always of the type $G_{a \bar{b} c \bar{d}}$ or $G_{a \bar{b} z \bar{z}}$. Contrarily to this, the flux appearing in the Dirac equation of the brane world-volume is always of type $G_{a \bar{b} c \bar{z}}$ or $G_{a \bar{b} z \bar{c}}$. Thus for $N=2$ flux, the Dirac equation does not change at all and the zero modes are same as those of the fluxless case.

We now turn our attention to fluxes preserving $N=1$ supersymmetry. Such a flux is of the form

$$
G_{4} \in\left(H^{2,0}\left(K 3_{1}\right) \otimes H^{0,2}\left(K 3_{2}\right)\right) \oplus\left(H^{0,2}\left(K 3_{1}\right) \otimes H^{2,0}\left(K 3_{2}\right)\right)
$$

In addition, it may contain flux of the form as given in Eq.(3.40). The susy conditions in presence of such a flux have been analyzed in great detail in [29]. It has been realized 
there, that by an appropriate choice of $(2,2)$ primitive flux, it is in fact possible to lift all the complex structure as well as Kähler moduli except the overall size of the $K 3$. It has also been noticed that the fluxes of the type given in eq.(3.41) stabilize both the $K 3 \mathrm{~s}$ at an attractor point [30]. Attractive $K 3$ surfaces are completely classified. They are in one-to-one correspondence with the $(\mathrm{SL}(2, \mathrm{Z})$ equivalent) matrices

$$
Q=\left(\begin{array}{cc}
2 a & b \\
b & 2 c
\end{array}\right)
$$

where $a, b$ and $c$ are integers, and in addition $a, c$ and the the determinant of $Q$ are required to be positive. Two such matrices represent the same $K 3$ if they are $S L(2, Z)$ equivalent. It has been shown in Ref.[30], that the tadpole cancellation condition puts very strong constraints on the integers $a, b$ and $c$ appearing in the above matrix $Q$. Thus the $N=1$ solutions are very limited and all of them can be determined.

We now consider $M 5$-branes wrapping divisors of the form $K 3 \times S$ in presence of such a flux. Locally, these fluxes are of the form $G_{a b \overline{c z}}, G_{a \overline{b c} z}$. The divisors under consideration have the cohomology

$$
H^{1,0}\left(K 3 \times P^{1}\right)=H^{3,0}\left(K 3 \times P^{1}\right)=0 .
$$

Since $\phi_{\bar{z}}$ and $\phi_{\bar{z} \bar{a} \bar{b}}$ belong to these cohomology groups, they must be identically zero. We can now clearly see from the Dirac equations that the forms $\phi, \phi \overline{a b}$ are harmonic, and in addition we have

$$
g^{a \bar{a}^{\prime}} g^{b \bar{b}^{\prime}} G_{a b \bar{c} \bar{z}} \phi_{\bar{a}^{\prime} \bar{b}^{\prime}}=0 .
$$

This condition lifts the $\phi_{\bar{a}^{\prime} \bar{b}^{\prime}}$ mode. Hence, we only have massless modes corresponding to $\phi \in H^{0,0}(D)$. Note, that all the spinors also carry an $S O(2,1)$-index, and hence there is a doubling of massless modes. Since $H^{0,0}(D)$ is one-dimensional, we are now left with two fermion zero modes, which is the right number for the instanton to contribute to $W_{n p}$.

We now study the Dirac equations in presence of $(3,1)-$ and $(4,0)-$ flux. They take the simple form

$$
\begin{aligned}
& \left(\partial_{\bar{c}} \phi+4 g^{b \bar{b}^{\prime}} \partial_{b} \phi_{\bar{b}^{\prime} \bar{c}}\right)=0, \\
& g^{a \bar{a}^{\prime}} g^{b \bar{b}^{\prime}} G_{a b \bar{c} \bar{z}} \phi_{\bar{a}^{\prime} \bar{b}^{\prime}}=0, \\
& \partial_{[\bar{a}} \phi_{\bar{b} \bar{c}]}=0 \\
& \phi G_{\bar{a} \bar{b} \bar{c} \bar{z}}=0
\end{aligned}
$$

Again, we find from the above that the forms $\phi, \phi \overline{a b}$ are harmonic. In addition, we find that both zero modes $\phi$ as well as $\phi_{\overline{a b}}$ must be zero. Thus the presence of $(4,0)$-flux 
lifts all the zero modes. As a result, we don't have any contribution to $W_{n p}$ from the M5-instantons.

We have seen in the above that we can choose an appropriate $(2,2)$ flux preserving $N=1$ susy, so that we have the correct number of fermion zero modes to have a non-perturbative superpotential. But once we include a $(4,0)$ flux, as enforced by the non-perturbative term in the susy conditions, all the zero modes are lifted which means that it is not consistent to keep the non-perturbative term. This raises a puzzle which we intend to resolve in the following section.

\section{Inclusion of the non-perturbative superpotential into the zero mode counting}

In the last section, we have seen that a $(4,0)$-component of $G$ lifts all zero modes. On the other hand, the susy conditions tell us that the $(4,0)$-part of $G$ is non-zero in the presence of $W_{n p}$. So there is an apparent mismatch. The resolution of this puzzle seems to be to include $W_{n p}$ into the Dirac equation which determines the number of zero modes. Then, $G_{4,0}$ should be balanced against $W_{n p}$, as it is the case for the susy conditions.

The Dirac part of the world-volume action on an $M 5$-brane with fluxes has the form $[8]$ :

$$
L_{f}^{M 5}=\frac{1}{2} \theta\left[\tilde{\gamma}^{m} \nabla_{m}+\frac{1}{24}\left(\gamma^{\hat{m} \hat{n} \hat{p}} \tilde{\gamma}^{q} G_{q \hat{m} \hat{n} \hat{p}}-\gamma^{\hat{q}} \tilde{\gamma}^{m n p} G_{m n p \hat{q}}\right)\right] \theta
$$

For us, it is important to note that the corresponding Dirac equation, whose solutions count the number of fermionic zero modes, is essentially determined by the susy variation of the 11-dimensional gravitino field. This can be seen as follows [11]. The supersymmetry conditions on the bulk, closed string background are given by

$$
\delta \psi_{M} \epsilon=0
$$

which is the supersymmetry transformation of the 11-dimensional gravitino. This can be translated to the linear part of the Dirac equation from the world-volume action as follows:

$$
\left(1-\Gamma_{M 5}\right) \Gamma^{\alpha} \delta \psi_{\alpha} \theta=0
$$

Here, $\delta \psi_{\alpha}$ is the pull-back of the gravitino variation to the brane via $\delta \psi_{\alpha}=\delta \psi_{M} \partial_{\alpha} x^{M}$ and $\Gamma_{\alpha}=\Gamma_{N} e_{M}^{N} \partial_{\alpha} x^{M}$. Therefore, one sees that the pull-back of the bulk gravitino 
equation is equivalent to a solution of the Dirac equation. Furthermore, one has to take into account the constraint from $\kappa$-symmetry on the $M 5$-brane:

$$
\left(1+\Gamma_{M 5}\right) \theta=0
$$

The number of zero modes is then given by the difference between the numbers of solutions of these two equations.

As we have already stated, we can recover the $M 5$-brane world-volume action eq.(4.45) by using the explicit expressions for the internal gravitino variations in the absence of $W_{n p}$ in eq.(4.47). We have already seen in $\S 2$ that turning on $W_{n p}$ alters the susy equations in the effective potential, as the effective superpotential now is $W=W_{f l u x}+W_{n p}$. This addition should be described by the modulino equations, i.e. $\delta \phi^{i}=\delta \phi^{I}=0$ should be now equivalent to $D W_{f l u x}+D W_{n p}=0$.

Substituting the expressions for the internal gravitino transformations with the general fluxes in (4.45), one obatins

$$
\begin{aligned}
& \left(\partial_{\bar{c}} \phi+4 g^{b \bar{b}^{\prime}} \partial_{b} \phi_{\bar{b}^{\prime} \bar{c}}\right)-2 g^{a \bar{a}^{\prime}} g^{b \bar{b}^{\prime}} g^{z \bar{z}} G_{a b \bar{c} z} \phi_{\bar{z} \bar{a}^{\prime} \bar{b}^{\prime}}=0, \\
& \left(\partial_{\bar{a}} \phi_{\bar{z}}+4 g^{b \bar{b}^{\prime}} \partial_{b} \phi_{\bar{z} \bar{a} \bar{b}^{\prime}}\right)+g^{a \bar{a}^{\prime}} g^{b \bar{b}^{\prime}} G_{a b \bar{c} \bar{z}} \phi_{\bar{a}^{\prime} \bar{b}^{\prime}}=0, \\
& \partial_{[\bar{a}} \phi_{\bar{b} \bar{c} \bar{c}}+\frac{1}{12} g^{z \bar{z}} \phi_{\bar{z}} G_{\bar{a} \bar{b} \bar{c} z}=0, \\
& \partial_{[\bar{a}} \phi_{\bar{z} \bar{b} \bar{c}]}+\frac{1}{24} \phi G_{\bar{a} \bar{b} \bar{c} \bar{z}}=0 .
\end{aligned}
$$

This is a set of local equations in the internal space. Every summand of (4.49) vanishes separately. This means that the set of equations

$$
\begin{aligned}
G_{a b \bar{c} z} \phi^{a b z} & =0, \\
G_{a b \overline{c z}} \phi^{a b} & =0, \\
G_{\overline{a b c z}} \phi^{z} & =0, \\
G_{\overline{a b c z}} \phi & =0
\end{aligned}
$$

is preventing the $\phi, \phi_{\bar{a}}, \phi_{\overline{a b}}$ and $\phi_{\overline{a b z}}$ to be non-trivail zero-modes in the case of general flux $G_{3}$. On the other hand $G_{m n p q}$ correspond to the three-dimensional constant scalar fields which one obtains as coefficients by expansion of $G_{3}$ in the harmonic basis on $C Y_{4}$ :

$$
\begin{aligned}
G_{4} & =G_{a b c d} d z^{a} \wedge d z^{b} \wedge d z^{c} \wedge d z^{d}+\sum_{i=1}^{h^{(3,1)}} G_{\bar{a} b c d}^{i} \omega^{i} \bar{a} b c d \\
& +\sum_{k=1}^{h^{(2,2)}} G_{\overline{a b c} d}^{k} \tilde{\omega}^{k} \overline{a b c d}+\sum_{i=1}^{h^{(3,1)}} G_{\overline{a b c} d^{i}} \bar{\omega}^{i} \overline{a b c} d \\
& +G_{\overline{a b c d}} d \bar{z}^{\bar{a}} \wedge d \bar{z}^{\bar{b}} \wedge d \bar{z}^{\bar{c}} \wedge d \bar{z}^{\bar{d}}
\end{aligned}
$$


with $\tilde{\omega}^{k}$ being basis elements of $H^{2,2}$. Since $H^{2,0}=0$, they can be expressed in terms of the basis elements $\omega^{I}$ of $H^{1,1}$ as $\tilde{\omega}^{k}=\sum_{I, J} \chi_{I J}^{k} \omega^{I} \wedge \omega^{J}$. The scalar fields $G, G^{k}, G^{i}$ are related to the flux superpotential by (2.22) and (2.23). From the modulino equations (2.27) we see that $W_{\text {flux }}$ has to be replaced by $W=W_{\text {flux }}+W_{\text {np }}$. This corresponds to the modification of $G$ to

$$
\begin{array}{ll}
\hat{G}_{2,2}: & \hat{G}_{a b \overline{c d}}=G_{a b \bar{c}}, \\
\hat{G}_{1,3}^{i}: & \hat{G}_{a \overline{b c d}}^{i}=\epsilon_{a \overline{b c d}} D_{i} W=G_{a \overline{b c d}}^{i}+\epsilon_{a \overline{b c d}} D_{i} W_{n p}, \\
\hat{G}_{0,4}: & \hat{G}_{\overline{a b c d}}=\epsilon_{\overline{a b c d}} \frac{D_{I} W}{\partial_{I} K}=G_{\overline{a b c d}}+\epsilon_{\overline{a b c d}} \frac{D_{I} W_{n p}}{\partial_{I} K} .
\end{array}
$$

This amounts to modifying the world volume action (4.45) in presenc of the nonperturbative superpotential, where we now replace $G$ by $\hat{G}$. It is than straightforward to see that, using the susy conditions $D_{i} W=D_{I} W=0$, the Dirac equation can be expressed as:

$$
\begin{aligned}
& \left(\partial_{\bar{c}} \phi+4 g^{b \bar{b}^{\prime}} \partial_{b} \phi_{\bar{b}^{\prime} \bar{c}}\right)=0, \\
& \left(\partial_{\bar{a}} \phi_{\bar{z}}+4 g^{b \bar{b}^{\prime}} \partial_{b} \phi_{\bar{z} \bar{a} \bar{b}^{\prime}}\right)+g^{a \bar{a}^{\prime}} g^{b \bar{b}^{\prime}} G_{a b \bar{c} \bar{z}} \phi_{\bar{a}^{\prime} \bar{b}^{\prime}}=0, \\
& \partial_{[\bar{a}} \phi_{\bar{b} \bar{c}]}=0, \\
& \partial_{[\bar{a} \bar{a}} \phi_{\bar{z} \bar{b} \bar{c}]}=0 .
\end{aligned}
$$

These conditions are identical to the ones coming from $(2,2)$ primitive flux without $W_{n p}$. The $(4,0)$ - and $(3,1)$ - parts of the flux are compensated by the nonperturbative term. As a result, we find that the number of fermion zero modes is unaltered. The apparent mismatch of the two answers in the previous section was due to the fact that we had then ignored the back-reaction of the instanton on the background flux and the geometry. Once we take care of this by modifying the fermionic terms accordingly, we obtain the expected result.

For the type II B Euclidean D3-brane, the story is very similar, hence we will be very brief in the following. The Dirac Lagrangian can be written in terms of the type $I I B$ gravitino variation, where in addition also the dilatino variation appears:

$$
L_{f}^{D 3}=\frac{1}{2} e^{-\phi} \sqrt{\operatorname{det} g} \bar{\theta}\left(1-\Gamma_{D 3}\right)\left(\Gamma^{\alpha} \delta \psi_{\alpha}-\delta \lambda\right) \theta,
$$

where the bulk susy variations are $\delta \psi_{m}=0$ and $\delta \lambda=0$. Substituting the expressions for $\delta \psi_{m}$ and $\delta \lambda$ without $W_{n p}$ into the above equation yields

$$
L^{D 3}=2 \sqrt{\operatorname{det} g} \theta\left\{e^{-\phi} \gamma^{m} \nabla_{m}+\frac{1}{8} \tilde{G}_{m n \hat{p}} \gamma^{m n \hat{p}}\right\} \theta .
$$

Once we use the modified expressions for $\delta \phi^{k}, \delta \phi^{i}, \delta \phi^{I}$ and $\delta \lambda$ in presence of $W_{n p}$, we replace $G$ by

$$
\hat{G}_{2,1}: \hat{G}_{a b \bar{c}}=\tilde{G}_{a b \bar{c}}
$$




$$
\begin{array}{ll}
\hat{G}_{1,2}^{i}: & \hat{G}_{a \overline{b c}}^{i}=\tilde{G}_{a \overline{b c}}^{i}+\epsilon_{a \overline{b c}} D_{i} W_{n p} \\
\hat{G}_{0,3} & : \quad \hat{G}_{\overline{a b c}}=\epsilon_{\overline{a b c}} \frac{D_{I} W}{\partial_{I} K}=\tilde{G}_{\overline{a b c}}+\epsilon_{\overline{a b c}} \frac{D_{I} W_{n p}}{\partial_{I} K} \\
\hat{G}_{3,0} & : \quad \hat{G}_{\overline{a b c}}=\epsilon_{a b c}(\bar{\tau}-\tau) D_{\tau} W=\tilde{G}_{\overline{a b c}}+\epsilon_{a b c}(\bar{\tau}-\tau) D_{I} W_{n p} .
\end{array}
$$

We can similarly analyze the Dirac equations. As expected, the number of fermion zero modes remains the same as in the case of primitive $(2,1)$-flux without the nonperturbative term.

\section{Acknowledgments}

We thank G. Cardoso, M. Haack, S. Mahapatra, S. Stieberger, S. Trivedi and M. Zagermann for useful discussion. S. Reffert and W. Schulgin thank the university of Munich for hospitality.

\section{A Dimensional reduction of $\delta \psi_{m}$}

We demonstrate the dimensional reduction of the supersymmetric variation of the gravitino on $C Y_{4}$.

Firstly, we write the internal gravitino variation using holomorphic and antiholomorphic indices.

$$
\begin{aligned}
& \delta \psi_{e}=\left[\nabla_{e}+\frac{1}{24}\left(3 \gamma^{b \overline{b d}} G_{e b \overline{c d}}+\gamma^{\overline{b c d}} G_{e \overline{b c d}}\right)\right] \xi \\
& \delta \psi_{\bar{e}}=\left[\nabla_{\bar{e}}+\frac{1}{24}\left(3 \gamma^{b \overline{b c d}} G_{\bar{e} b \overline{c d}}+\gamma^{\overline{b c d}} G_{\overline{e b c d}}\right)\right] \xi
\end{aligned}
$$

$\psi_{m}$ is a vector-spinor, where $m$ is an internal vector index which transforms in the $\mathbf{4} \oplus \overline{\mathbf{4}}$ representation of $S U(4)$. The spinor index of the eleven dimensional gravitino transforms in the $\mathbf{3 2}$ under $S O(1,10)$. After compactification on a $C Y_{4}, S O(1,10)$ is broken to $S U(4) \times S O(2,1)$ and the spinor transforms in the $(\mathbf{1}, \mathbf{2}) \oplus(\mathbf{4}, \mathbf{2}) \oplus(\mathbf{6}, \mathbf{2}) \oplus(\overline{\mathbf{4}}, \mathbf{2}) \oplus(\overline{\mathbf{1}}, \mathbf{2})$. This means that $\psi_{e}$ can be written as a sum of $(0, p)$-forms with one additional holomorphic or antiholomorphic index.

$$
\psi_{e}=\phi_{e}\left|\Omega>+\phi_{e \bar{a}} \gamma^{\bar{a}}\right| \Omega>+\phi_{e \overline{a b}} \gamma^{\overline{a b}}\left|\Omega>+\phi_{e \overline{a b c}} \gamma^{\overline{a b c}}\right| \Omega>+\phi_{e \overline{a b c d}} \gamma^{\overline{a b c d}} \mid \Omega>
$$

Note that $\psi_{e}$ in (A.58) has an additional spinor index which transforms in the $\mathbf{2}$ of $S O(1,2)$. The rhs. of $(\mathrm{A} .57)$ is also such a spinor. $\xi$ can be written as $\xi=\epsilon \otimes \eta$, where $\eta$ is a covariantly constant spinor on the $C Y_{4}$ and $\epsilon$ a supersymmetry parameter in the non-compact dimensions. We write $\xi$ as

$$
\xi=\hat{\xi}\left|\Omega>+\hat{\xi} \overline{a b c d} \gamma^{\overline{a b c d}}\right| \Omega>
$$


and should remember that $\hat{\xi}$ has an additional index which transforms in the $\mathbf{2}$ under $S O(1,2)$. The rhs. of the first equation in $(\mathrm{A} .57)$ is then

$$
\begin{aligned}
\delta \psi_{e} & =\left[\nabla_{e}+\frac{1}{24}\left(3 \gamma^{b \overline{c d}} G_{e b \overline{c d}}+\gamma^{\overline{b c d}} G_{e \overline{b c d}}\right)\right] \xi \\
& =\left[\nabla_{e}+\frac{1}{24}\left(6 G_{e b \overline{c d}} g^{b \bar{d}} \gamma^{\bar{c}}+G_{e \overline{b c d}} \gamma^{\overline{b c d}}\right)\right] \xi \\
& =\nabla_{e}\left(\hat{\xi}\left|\Omega>+\hat{\xi}_{\overline{a b c d}} \gamma^{\overline{a b c d}}\right| \Omega>\right)+\frac{1}{4} G_{e b \bar{c}} g^{b \bar{d}} \hat{\xi} \gamma^{\bar{c}}\left|\Omega>+\frac{1}{24} G_{e \overline{b c d}} \hat{\xi} \gamma^{\overline{b c d}}\right| \Omega>(
\end{aligned}
$$

The open index $e$ corresponds to a one-form index, which means that we have a collection of $(1, p)$-forms. ${ }^{5}$ We compare the forms of the same type on both sides and obtain the following set of equations:

$$
\begin{aligned}
\delta\left(\phi_{e \bar{a}} \gamma^{\bar{a}} \mid \Omega>\right) & =\frac{1}{4} G_{e b \bar{c}} g^{b \bar{d}} \hat{\xi} \gamma^{\bar{c}} \mid \Omega>, \\
\delta\left(\phi_{e a b} \gamma^{\overline{a b}} \mid \Omega>\right) & =0, \\
\delta\left(\phi_{e \overline{a b c}} \gamma^{\overline{a b c}} \mid \Omega>\right) & =\frac{1}{24} G_{e \overline{b c d}} \hat{\xi} \gamma^{\overline{b c d}} \mid \Omega>.
\end{aligned}
$$

These are the only forms from (A.60), which do not vanish on a $C Y_{4}$.

Let us look at the second equation of (A.57) where the additional index is antiholomorphic. To see this index as a form index we have to make it holomorphic. This can be done by applying Serre's generalization of Poincaré duality

$$
\tilde{\psi}_{a b c}=\psi_{\bar{e}} g^{e \bar{e}} \omega_{a b c e}
$$

where $\omega_{a b c e}$ is the $(4,0)$-form of the $C Y_{4}$.

$$
\begin{aligned}
\delta \tilde{\psi}_{a b c} & =g^{e \bar{e}} \omega_{a b c e}\left(\nabla_{\bar{e}}+\frac{1}{24}\left(3 \gamma^{f \overline{g h}} G_{\overline{e f} \overline{g h}}+\gamma^{\overline{f g h}} G_{\overline{e f g h}}\right)\right)\left(\hat{\xi} \mid \Omega>+\hat{\xi}_{\overline{i j k l}} \gamma^{\overline{i j k l}}\right) \mid \Omega> \\
& =g^{e \bar{e}} \omega_{a b c e}\left(\nabla_{\bar{e}}+\frac{1}{4} G_{\bar{e} f \overline{f h}} g^{f \bar{h}} \gamma^{\bar{g}}+\frac{1}{24} G_{\overline{e f g h}} \gamma^{f g h}\right)\left(\hat{\xi} \mid \Omega>+\hat{\xi}_{\overline{i j k l}} \gamma^{\overline{i j k l}}\right) \mid \Omega>\mathrm{A}
\end{aligned}
$$

Again, comparing the forms of the same type gives us

$$
\begin{aligned}
\delta\left(\tilde{\phi}_{a b c \bar{a}} \gamma^{\bar{a}}\right) \mid \Omega> & =\frac{1}{4} g^{e \bar{e}} \omega_{a b c e} G_{\bar{e} f \overline{g h}} g^{f \bar{h}} \gamma^{\bar{g}} \hat{\xi} \mid \Omega>, \\
\delta\left(\tilde{\phi}_{a b c \overline{a b}} \gamma^{\overline{a b}}\right) \mid \Omega> & =0, \\
\delta\left(\tilde{\phi}_{a b c \overline{a b c}} \gamma^{\overline{a b c}}\right) \mid \Omega> & =\frac{1}{24} g^{e \bar{e}} \omega_{a b c e} G_{\overline{e f g h}} \gamma^{\overline{f g h}} \hat{\xi} \mid \Omega>.
\end{aligned}
$$

Eqs. (A.61) and (A.64) can be expanded in the basis of harmonic forms on the $C Y_{4}$ as follows:

$$
\delta\left(\phi_{i} \omega_{(1,3)}^{i}\right)=g_{i} \omega_{(1,3)}^{i} \hat{\xi}
$$

\footnotetext{
${ }^{5}$ We can introduce a second set of gamma matrices, which will commute with the first one, so for example $\phi_{a_{1} \ldots a_{p} \bar{a}_{1} \ldots \bar{a}_{q}} \tilde{\gamma}^{a_{1}} \ldots \tilde{\gamma}^{a_{p}} \gamma^{\bar{a}_{1}} \ldots \gamma^{\bar{a}_{q}} \mid \Omega>$ will correspond to a $(p, q)$-form. Here we will omit the second set of gamma-matrices to make the equations more transparent. A detailed explanation of this formalism is given in Chapter 15 of [31].
} 


$$
\begin{aligned}
\delta\left(\phi_{I} \omega_{(1,1)}^{I}\right) & =g_{I} \omega_{(1,1)}^{I} \hat{\xi} \\
\delta\left(\phi_{i} \omega_{(1,2)}^{i}\right) & =0 \\
\delta\left(\phi_{i} \omega_{(2,3)}^{i}\right) & =0
\end{aligned}
$$

where $\omega_{(1,1)}^{I}$ and $\omega_{(1,3)}^{i}$ are basis elements of $H^{1,1}\left(C Y_{4}\right)$ and $H^{1,3}\left(C Y_{4}\right)$ respectively.

If we repeat the calculations for the type IIB case, we obtain an equation for the $(1,2)$-form, another one for the $(2,2)$-form and $(3,0)$-form for the dilatino:

$$
\begin{aligned}
\delta\left(\phi_{e \overline{a b}} \gamma^{\overline{a b}} \mid \Omega>\right) & =-\frac{1}{8} G_{e \overline{a b}} \gamma^{\overline{a b}} \hat{\xi}^{*}\left|\Omega>-\frac{1}{16} g^{a \bar{c}} g_{e \bar{a}} G_{a \overline{a c}} \gamma^{\overline{a b}} \hat{\xi}^{*}\right| \Omega>, \\
\delta\left(\phi_{\bar{e} \mid \overline{a b}} \gamma^{\overline{a b}} \mid \Omega>\right) & =-\frac{1}{16} G_{\overline{a b e}} \gamma^{\overline{a b}} \hat{\xi}^{*} \mid \Omega>, \\
\delta\left(\lambda_{\overline{a b c}}^{*} \gamma^{\overline{a b c}} \mid \Omega>\right) & =\frac{i}{4} \bar{G} \overline{a b c} \gamma^{\overline{a b c}} \hat{\xi}^{*} \mid \Omega>.
\end{aligned}
$$

The second equation corresponds to the $(2,2)$-form ${ }^{6}$ after applying Serre's duality and to a $(1,1)$-form by forming the Hodge dual.

These equations (A.66) can be expanded in the basis of harmonic forms on the $C Y_{3}$ and written then as

$$
\begin{array}{rlrl}
\delta\left(\phi_{i} \omega_{(1,2)}^{i}\right) & =g_{i} \omega_{(1,2)}^{i} \hat{\xi}, & & i=1, \ldots, h^{(2,1)}, \\
\delta\left(\phi_{I} \omega_{(1,1)}^{I}\right) & =g_{I} \omega_{(1,1)}^{I} \hat{\xi}, & & I=1, \ldots, h^{(1,1)}, \\
\delta\left(\lambda^{(0,3)} \omega_{(0,3)}\right) & =g^{(0,3)} \omega_{(0,3)} \hat{\xi} . &
\end{array}
$$

$\phi_{i}, \phi_{I}$ and $\lambda^{(0,3)}$ correspond to the 4-dimensional complex structure modulinos, the Kähler modulinos and the dilatino respectively.

Finally, let us rewrite the variation of the modulino fields as it will be needed for our investigation:

For the M-theory case:

$$
\begin{aligned}
\delta \phi_{e \bar{c}}^{k} & =\frac{1}{4}\left(G_{e b \overline{c d}} g^{b \bar{d}}\right)^{k} \hat{\xi}, & k & =1, \ldots, h^{(1,1)}, \\
\delta \phi_{e \overline{a b c}}^{i} & =\frac{1}{24} G_{e \overline{a b c}}^{i} \hat{\xi}, & i & =1, \ldots, h^{(3,1)}, \\
\delta \phi_{\bar{e} \mid \overline{a b c}}^{I} & =\frac{1}{24} G_{\overline{e a b c} \hat{\xi}} \hat{\xi}, & I & =1, \ldots, h^{(1,1)} .
\end{aligned}
$$

For the type IIB case

$$
\delta \phi_{e \overline{a b}}^{i}=-\frac{1}{8} G_{e \overline{a b}}^{i} \hat{\xi}^{*}-\frac{1}{16} g^{a \bar{c}} g_{e \bar{a}} G_{a \overline{b c}}^{i} \hat{\xi}^{*}, \quad i=1, \ldots, h^{(2,1)}
$$

\footnotetext{
${ }^{6}$ Note, that in this notation the holomorphic indices correspond to the holomorphic part of the form and vice versa. The antiholomorphic index $\bar{e}$ has no meaning as form index before applying Serre's duality. That is why we put $\mid$ there to prevent its mixing with the antiholomorphic indices.
} 


$$
\begin{aligned}
\delta \phi_{\bar{e} \mid \overline{a b}}^{I} & =-\frac{1}{16} G \overline{\overline{e a b}} \hat{\xi}^{*}, & I=1, \ldots, h^{(1,1)} \\
\delta \lambda_{\overline{a b c}}^{*} & =\frac{i}{4} \bar{G} \overline{a b c} \hat{\xi}^{*} . &
\end{aligned}
$$

We label the modulinos with the indices $k, i, I$. Additionally, they have indices from the beginning of the alphabet. Let us briefly comment about this.

A $(p, q)$-form $\nu$ can be expanded in the basis of harmonic $(p, q)$-forms $\omega^{i}: \nu=\nu_{i} \omega^{i}$. In the case of a complex manifold, the number of the harmonic forms is given by the corresponding Hodge number $h^{(p, q)}$. On the other hand we can write the form in every local patch as $\nu=\nu_{a_{1} \ldots a_{p}} \bar{a}_{1} \ldots \bar{a}_{q} d z^{a_{1}} \wedge \ldots \wedge d z^{a_{p}} \wedge d z^{\bar{a}_{1}} \wedge \ldots \wedge d z^{\bar{a}_{q}}$. If $\nu_{a_{1} \ldots a_{p}} \bar{a}_{1} \ldots \bar{a}_{q}$ are constant, they should correspond to the coefficients $\nu_{i}$. The whole $\nu_{a_{1} \ldots a_{p}} \bar{a}_{1} \ldots \bar{a}_{q}$ in all coordinate patches span a vector space, in which so many $\nu_{a_{1} \ldots a_{p}} \bar{a}_{1} \ldots \bar{a}_{q}$ are linearly dependent by the transition functions that the dimension of this vector space is $h^{(p, q)}$. The linearly

independent combinations of $\nu_{a_{1} \ldots a_{p}} \bar{a}_{1} \ldots \bar{a}_{q}$ are then in one to one correspondence to the $\nu_{i}$.

\section{References}

[1] S. Kachru, R. Kallosh, A. Linde and S. P. Trivedi, "De Sitter vacua in string theory," Phys. Rev. D 68, 046005 (2003) [arXiv:hep-th/0301240].

[2] F. Denef, M. R. Douglas and B. Florea, "Building a better racetrack," JHEP 0406, 034 (2004) [arXiv:hep-th/0404257].

[3] F. Denef, M. R. Douglas, B. Florea, A. Grassi and S. Kachru, "Fixing all moduli in a simple F-theory compactification," arXiv:hep-th/0503124.

[4] E. Witten, "Non-Perturbative Superpotentials In String Theory," Nucl. Phys. B 474, 343 (1996) [arXiv:hep-th/9604030].

[5] R. Kallosh and D. Sorokin, "Dirac action on M5 and M2 branes with bulk fluxes," JHEP 0505, 005 (2005) [arXiv:hep-th/0501081].

[6] P. K. Tripathy and S. P. Trivedi, "D3 brane action and fermion zero modes in presence of background flux," JHEP 0506, 066 (2005) [arXiv:hep-th/0503072].

[7] N. Saulina, "Topological constraints on stabilized flux vacua," Nucl. Phys. B 720, 203 (2005) [arXiv:hep-th/0503125]. 
[8] R. Kallosh, A. K. Kashani-Poor and A. Tomasiello, "Counting fermionic zero modes on M5 with fluxes," JHEP 0506, 069 (2005) [arXiv:hep-th/0503138].

[9] L. Martucci, J. Rosseel, D. Van den Bleeken and A. Van Proeyen, "Dirac actions for D-branes on backgrounds with fluxes," Class. Quant. Grav. 22, 2745 (2005) [arXiv:hep-th/0504041].

[10] P. Berglund and P. Mayr, "Non-perturbative superpotentials in F-theory and string duality," arXiv:hep-th/0504058.

[11] E. Bergshoeff, R. Kallosh, A. K. Kashani-Poor, D. Sorokin and A. Tomasiello, "An index for the Dirac operator on D3 branes with background fluxes," arXiv:hepth/0507069.

[12] J. Park, "D3 instantons in Calabi-Yau orientifolds with(out) fluxes," arXiv:hepth/0507091.

[13] J. Gomis, F. Marchesano and D. Mateos, "An open string landscape," arXiv:hepth/0506179.

[14] G. Curio, A. Krause and D. Lüst, "Moduli stabilization in the heterotic/IIB discretuum," arXiv:hep-th/0502168.

[15] D. Lüst, S. Reffert, W. Schulgin and S. Stieberger, "Moduli stabilization in type IIB orientifolds. I: Orbifold limits," arXiv:hep-th/0506090.

[16] T. R. Taylor and C. Vafa, "RR flux on Calabi-Yau and partial supersymmetry breaking," Phys. Lett. B 474, 130 (2000) [arXiv:hep-th/9912152].

[17] S. B. Giddings, S. Kachru and J. Polchinski, "Hierarchies from fluxes in string compactifications," Phys. Rev. D 66, 106006 (2002) [arXiv:hep-th/0105097].

[18] S. Gukov, C. Vafa and E. Witten, "CFT's from Calabi-Yau four-folds," Nucl. Phys. B 584, 69 (2000) [Erratum-ibid. B 608, 477 (2001)] [arXiv:hep-th/9906070].

[19] K. Becker and M. Becker, "M-Theory on Eight-Manifolds," Nucl. Phys. B 477, 155 (1996) [arXiv:hep-th/9605053].

[20] S. Kachru, M. B. Schulz and S. Trivedi, "Moduli stabilization from fluxes in a simple IIB orientifold," JHEP 0310, 007 (2003) [arXiv:hep-th/0201028].

[21] M. Grana and J. Polchinski, "Gauge / gravity duals with holomorphic dilaton," Phys. Rev. D 65, 126005 (2002) [arXiv:hep-th/0106014]. 
[22] G. L. Cardoso, G. Curio, G. Dall'Agata and D. Lüst, "BPS action and superpotential for heterotic string compactifications with JHEP 0310, 004 (2003) [arXiv:hepth/0306088].

[23] D. Robbins and S. Sethi, "A barren landscape," Phys. Rev. D 71, 046008 (2005) [arXiv:hep-th/0405011].

[24] L. Görlich, S. Kachru, P. K. Tripathy and S. P. Trivedi, "Gaugino condensation and nonperturbative superpotentials in flux compactifications," JHEP 0412, 074 (2004) [arXiv:hep-th/0407130].

[25] M. Grana, "D3-brane action in a supergravity background: The fermionic story," Phys. Rev. D 66, 045014 (2002) [arXiv:hep-th/0202118].

[26] D. Marolf, L. Martucci and P. J. Silva, "Fermions, T-duality and effective actions for D-branes in bosonic backgrounds," JHEP 0304, 051 (2003) [arXiv:hep-th/0303209].

[27] D. Marolf, L. Martucci and P. J. Silva, "Actions and fermionic symmetries for Dbranes in bosonic backgrounds," JHEP 0307, 019 (2003) [arXiv:hep-th/0306066].

[28] P. S. Aspinwall, “K3 surfaces and string duality,” arXiv:hep-th/9611137.

[29] D. Lüst, P. Mayr, S. Reffert and S. Stieberger, "F-theory flux, destabilization of orientifolds and soft terms on D7-branes," arXiv:hep-th/0501139.

[30] P. S. Aspinwall and R. Kallosh, "Fixing all moduli for M-theory on K3 x K3," arXiv:hep-th/0506014.

[31] M. B. Green, J. H. Schwarz and E. Witten, "Superstring Theory. Vol. 2: Loop Amplitudes, Anomalies And Phenomenology," 